\title{
Anomalies in the Counts of Low Redshift Quasars
}

\author{
D. P. Youll \\ Basingstoke, UK \\ Email: david.youll@hotmail.com
}

How to cite this paper: Youll, D.P. (2021) Anomalies in the Counts of Low Redshift Quasars. Journal of High Energy Physics, Gravitation and Cosmology, 7, 531-550. https://doi.org/10.4236/jhepgc.2021.72030

Received: January 29, 2021

Accepted: April 17, 2021

Published: April 20, 2021

Copyright (c) 2021 by author(s) and Scientific Research Publishing Inc. This work is licensed under the Creative Commons Attribution International License (CC BY 4.0).

http://creativecommons.org/licenses/by/4.0/

\begin{abstract}
The commonly held view by astronomers and cosmologists is that redshift indicates distance for both galaxies and quasars. However, data from over 750,000 quasars, and specifically about 9500 with high accuracy measurements in the redshift range 0.05 to 0.25 , contained in the Sloan Digital Sky Survey (SDSS) data release DR16 indicates several anomalies that might indicate that many quasars do not follow the same redshift-distance relationship as measured for galaxies. Also, while the commonly held view is that quasars are, in general, much brighter than galaxies, in the redshift range up to 0.5, this is not the case in the nearby universe. Two proposals are given that might explain why quasars do not follow the same redshift-distance relationship as measured for galaxies and why they might be fainter than galaxies at lower redshifts. Astronomers are urged to find more quasars in the redshift range 0 $<Z<1.0$ in order to clarify the luminosity distribution function of quasars at low redshift.
\end{abstract}

\section{Keywords}

Quasar, Redshift, SDSS

\section{Introduction}

Following the work of Hubble and others almost a century ago, a redshift-distance relationship is now commonly accepted to apply to galaxies and supernovae up to redshift of at least $Z=1.0$. In addition, most studies that involve the measured redshift of quasars assume that the redshift of every quasar is a direct indication of their distance. However, this paper presents an analysis of data from the Sloan Digital Sky Survey data release DR16 [1] that shows that, while many quasars exhibit a redshift-distance relationship, that relationship is not the same as that seen for galaxies and supernovae. 
The Sloan Digital Sky Survey, Data Release 16, contains over 750,000 quasars with luminosity and redshift measurements. This paper investigates the redshift-distance relationship for quasars using the statistics that such a large volume of data enables. This analysis is based on the premises that:

- the density of quasars in the universe is constant, at least in the redshift range $0<Z<0.25$ where the main analysis in this paper is performed;

- at any specific distance,

- quasars will have the same cosmological redshift,

- there will be a range of quasar luminosity and the count of quasars at each luminosity will exhibit a similar profile at different distances (see analysis and Appendix A for more details);

- at further distances,

- the count of quasars will increase with distance as the spherical shell associated with a redshift increment increases (assuming a redshift-distance relationship based on an expanding universe, see Appendix A),

- the count of quasars at low luminosity will be lower than expected due to the technical constraints in detecting low luminosity objects.

There are factors that may affect the analysis:

- quasars move in relation to each other, so quasars at a particular distance will have redshifts that reflect the impact of their intrinsic peculiar motion. As there is no reason to expect quasars to have a higher intrinsic motion than that found for galaxies, this factor is not expected to have significant impact on the results.

- the quasars contained in the Sloan Digital Sky Survey (SDSS) DR16 have been selected (see [1] for more information) according to colour, redshift and other factors. Hence, the result of these selections is that the quasars in DR16 do not represent the full population of quasars in the universe, see Appendix B for more information on this topic.

- a variety of telescopes, instruments and analysis techniques have been used to collect data. Each of these has its own limitations (see [1] for more information) and these limitations are seen in the data counts because specific combinations of telescopes, instruments and analysis techniques have been used to detect quasars at different luminosities and redshifts. For example, the larger telescopes have mainly been used to detect fainter quasars and some instruments are only used for surveys of specific redshift ranges.

\section{Approach Taken}

Data was downloaded from the Sloan Digital Sky Survey DR16 data release (available via https://apps.sciserver.org/login-portal/) using the following query: select

objid, ra, dec, psfmag_u, psfmag_g, psfmag_r, psfmag_i, psfmag_z, Z, psfMagErr_u, psfMagErr_g, psfMagErr_r, psfMagErr_i, psfMagErr_z, Zerr, class from SpecPhoto where class='QSO' 
Notes:

- RA, Dec are J2000 Right Ascension and Declination.

- Luminosity is measured in magnitudes (psfmag_u, etc), through filters (colours). As PSF magnitudes are optimal measures of the fluxes of stars and these measurements are recommended by SDSS for use with quasars, they are used throughout this paper. The filters used by SDSS are green (g), red (r), and three colours that correspond to light not visible to the human eye: ultraviolet $(\mathrm{u})$, and two infrared wavelengths ( $\mathrm{i}$ and $\mathrm{z}$ ). For more info: https://www.sdss.org/dr12/algorithms/magnitudes/.

- Accuracy/error in each luminosity measurement is provided by psfMagERR_u, etc.

- $Z$ is the measured redshift.

- Zerr gives the accuracy/error of each redshift measurement.

- Class = "QSO" identifies the SDSS classification of an object as a quasar.

- For guidance on measures of flux from quasars and the means by which magnitude measure are made, see http://www.sdss3.org/dr8/algorithms/magnitudes.php.

- More information about the SDSS survey techniques and processes can be found in [2] Stoughton et al. 2002 (AJ 123:485-548).

The SDSS survey provides measures of the light flux from quasars through 5 filters as listed in Table 1 . These filters are referred to in lower case to avoid confusion between the filter " $z$ " and references to redshift, where " $Z$ " (i.e. upper case) is used. Figure 1 indicates the coverage of each filter and how the measure of the flux from a quasar through these filters is affected by increasing measured redshift (i.e. when the light is redshifted, the peak of the spectrum can appear in a different filter range than that of a quasar at zero redshift).

Data from SDSS DR16 data release was loaded into a Microsoft Access database, where a pivot table was used to create tables that were transferred to $\mathrm{Mi}$ crosoft Excel where charts were created. Magnitudes were rounded down to an accuracy of 0.1 (over $40 \%$ of quasar measurements have magnitude accuracy better than 0.1 and over $85 \%$ have magnitude accuracy better than 0.3 ) and measured redshifts $(Z$ ) were rounded down to an accuracy of 0.01 (over $99 \%$ of redshift measurements had an accuracy of less than 0.01). Error levels associated with the $\mathrm{u}$ and $\mathrm{z}$ filters are shown in Figure 2 (note the $\mathrm{u}$ filter generated the faintest measures and $\mathrm{z}$ the brightest measures).

Table 1. Colour filters.

\begin{tabular}{cccc}
\hline $\begin{array}{c}\text { Filter } \\
\text { Letter }\end{array}$ & Effective Wavelength Midpoint & $\begin{array}{c}\text { Full Width Half Maximum } \\
\text { (Bandwidth } \Delta \lambda \text { ) }\end{array}$ & Description \\
\hline $\mathrm{u}$ & $365 \mathrm{~nm}$ & $66 \mathrm{~nm}$ & Ultraviolet \\
$\mathrm{g}$ & $464 \mathrm{~nm}$ & $128 \mathrm{~nm}$ & Green \\
$\mathrm{r}$ & $658 \mathrm{~nm}$ & $138 \mathrm{~nm}$ & Red \\
$\mathrm{i}$ & $806 \mathrm{~nm}$ & $149 \mathrm{~nm}$ & Infrared \\
$\mathrm{z}$ & $900 \mathrm{~nm}$ & & \\
\hline
\end{tabular}




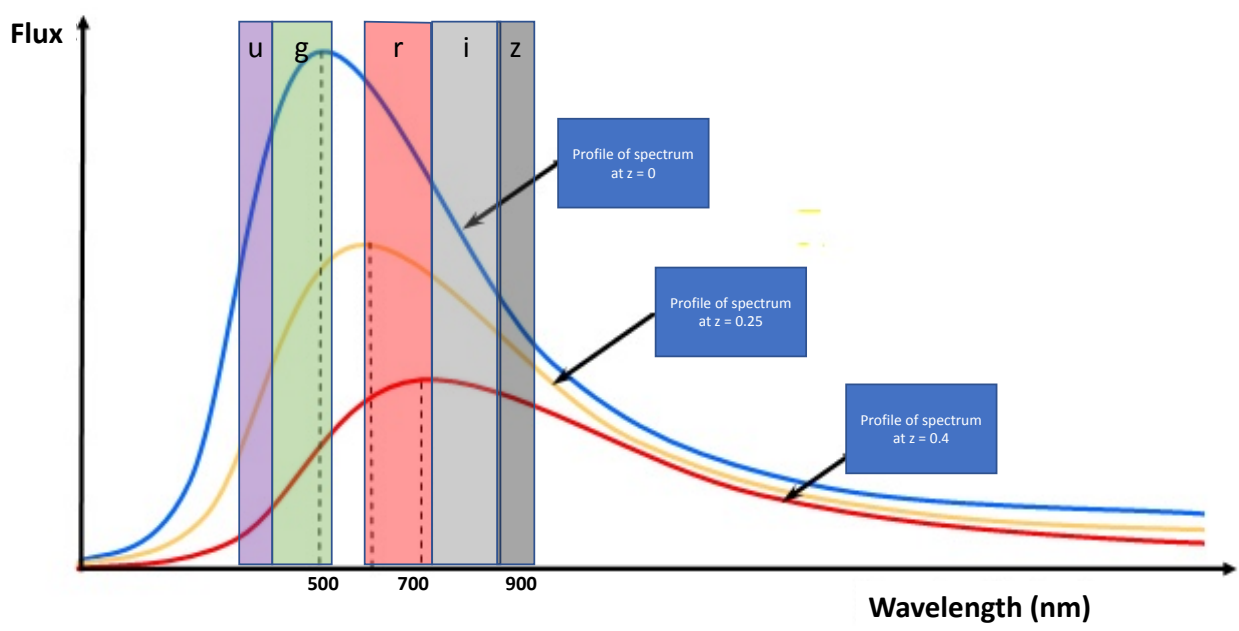

Figure 1. Effect of redshift on spectrum and flux measures through filters.

\section{Error levels associated with psfmag_u}

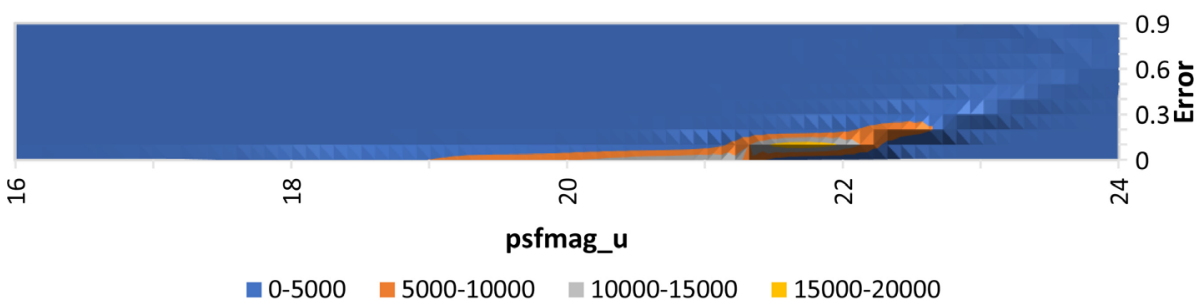

\section{Error levels associated with psfmag_z}

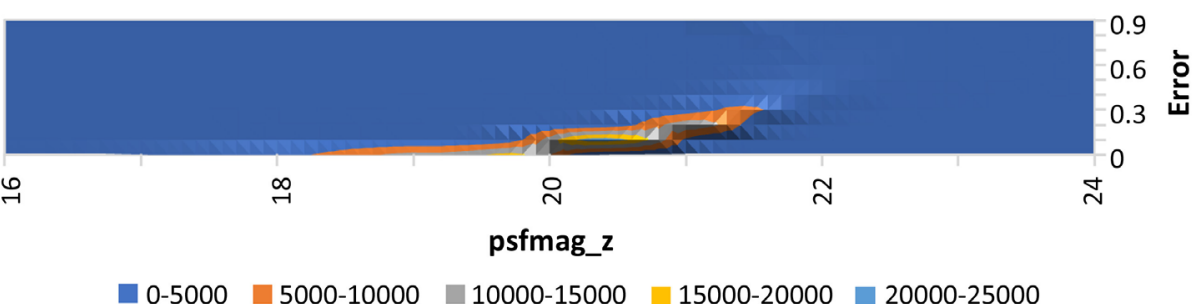

Figure 2. Accuracy/error associated with psfmag measures.

The counts of quasars from DR16 with low error in luminosity $(<0.1 \mathrm{mag})$ against redshift in bins of 0.01 in redshift is shown in Figure 3 (total 244,000 quasars). The count at any redshift is dependent on the survey techniques used to find and measure quasars at that redshift e.g. the peak in counts seen at about redshift 2.3 originates from one survey that was searching for quasars in the redshift range 2 to 2.5 .

There is a drop in count of quasars against magnitude (in magnitude bins of 0.1) across all measured redshifts (see example chart in Figure 4) at the following levels which are understood to be caused by SDSS quasar detection and selection techniques:

- psfmag_z $=19.1$ (the counts appear to be low across the range 18.9 to 19.8 see Figure 6), 


\section{Count of quasars against redshift}

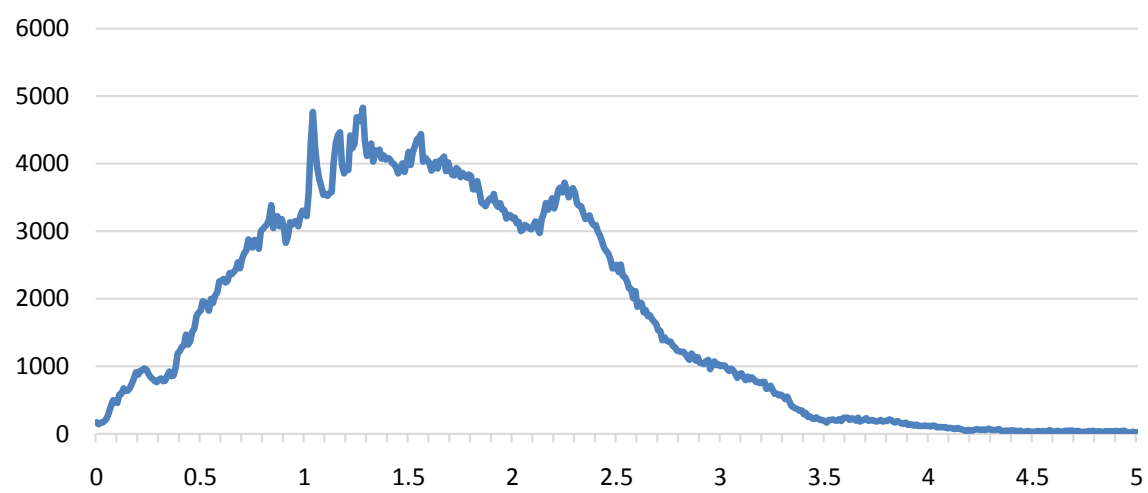

Figure 3. Count of quasars against redshift.

\section{Count v. psfmag_z}

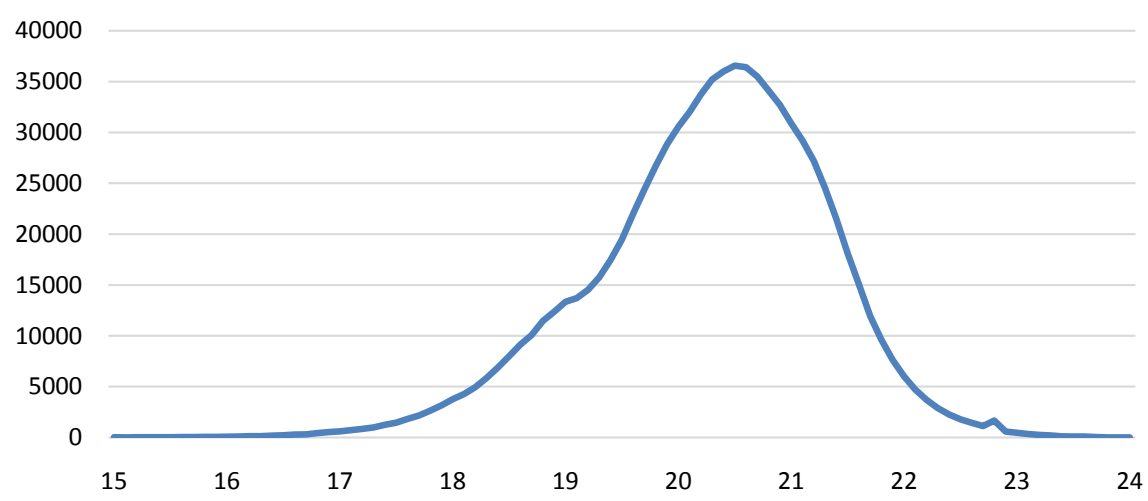

Figure 4. Count of quasars related to psfmag_z.

- psfmag_ $\mathrm{i}=19.2$ (the counts appear to be low across the range 19.0 to 20.2),

- psfmag_r $=19.4$ (the counts appear to be low across the range 19.0 to 20.0),

- psfmag_g = 19.6 (the counts appear to be low across the range 19.3 to 20.5).

- While there is not a drop shown in the psfmag_u counts, there is a significant step in the counts shown around psfmag_u $=19.7$ (the counts appear to be low across the range 19.3 to 20.3 ).

$K$-corrections ([3] [4] [5] [6]) are not applied in this version of the paper. The $K$-correction is defined as

$$
m=M+D M+K
$$

where $m$ is the observed apparent magnitude, $M$ is the absolute magnitude, $D M$ is the distance modulus. $D M=5 \log \left(D_{L} / 10 \mathrm{pc}\right)$ and $D_{L}$ is the luminosity distance. Quasar $K$-corrections have not been made in this paper because, it is understood that quasar $K$-corrections in the optical bands in the redshift range 0.05 to 0.25 where the main analysis is performed are not significant. A good approximation for the $K$ correction is $+1.25 \log (1+z)$ (at $z=0.2$ the $K$-correction is +0.099 , i.e. the luminosity is fainter than if no $K$-correction is applied), which indicates quasar $K$-corrections will be similar in the redshift range of this analysis and their impact will be within the error given. 


\section{Analysis}

An earlier paper ([7]) recorded several features associated with quasar counts. This paper focuses on the counts using data from quasars with low error in their measured/apparent magnitude and redshift measurements (0.1 and 0.001 maximum error respectively) in the measured redshift range 0 to 0.25 . Data for about 9500 quasars are in this redshift range. The quasar counts are shown in a chart (see Figure 5 left side), alongside a chart (see Figure 5 right side) showing galaxy counts from galaxy data in the SDSS DR14 data release (see [8] for more information). Counts in the redshift range up to 0.5 are shown for context. Dotted lines have been added to show the expected redshift-distance relationship for an object with a specific absolute magnitude (see details below). These were calculated using the following formula ([9]) i.e. redshift is assumed to originate from a velocity of recession):

$$
\text { Distance }=c *\left(\left((Z+1)^{2}-1\right) /\left((Z+1)^{2}+1\right)\right) / H
$$

where $c$ is the velocity of light, $Z$ is the true redshift of the quasar and $H$ is Hubble's constant (70 km/sec/Megaparsec).

These counts of quasars against redshift and measured luminosity indicate that:

- the redshift-distance relationship that is seen for galaxies is not seen for quasars and

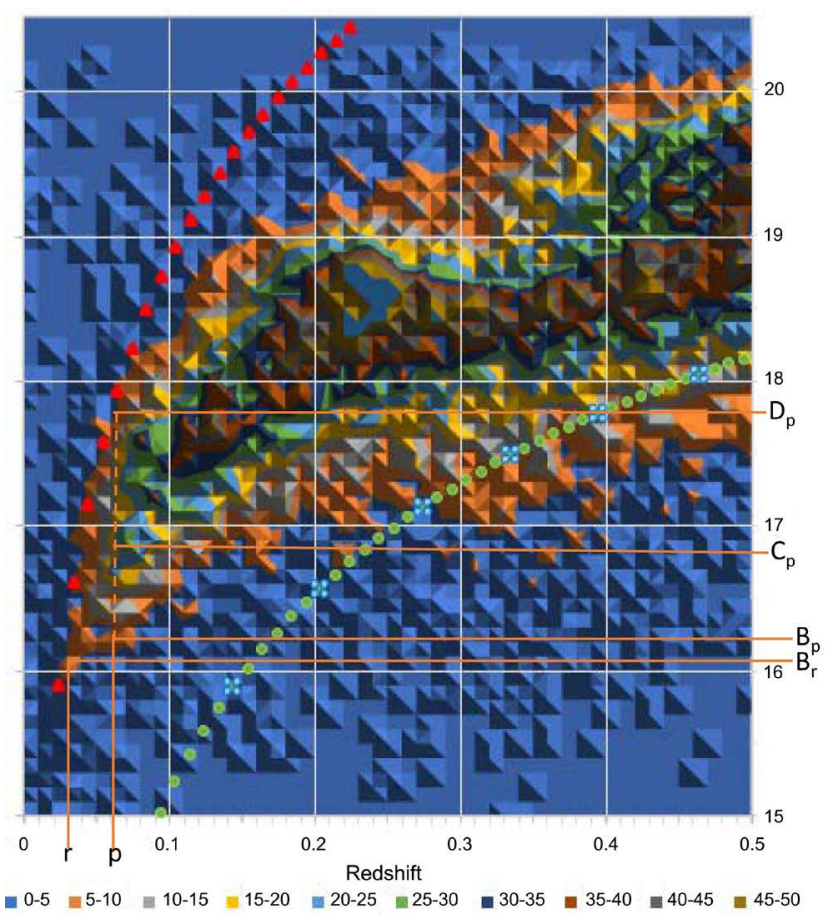

= $0-5=5-10=10-15=15-20=20-25=25-30=30-35=35-40=40-45=45-50$

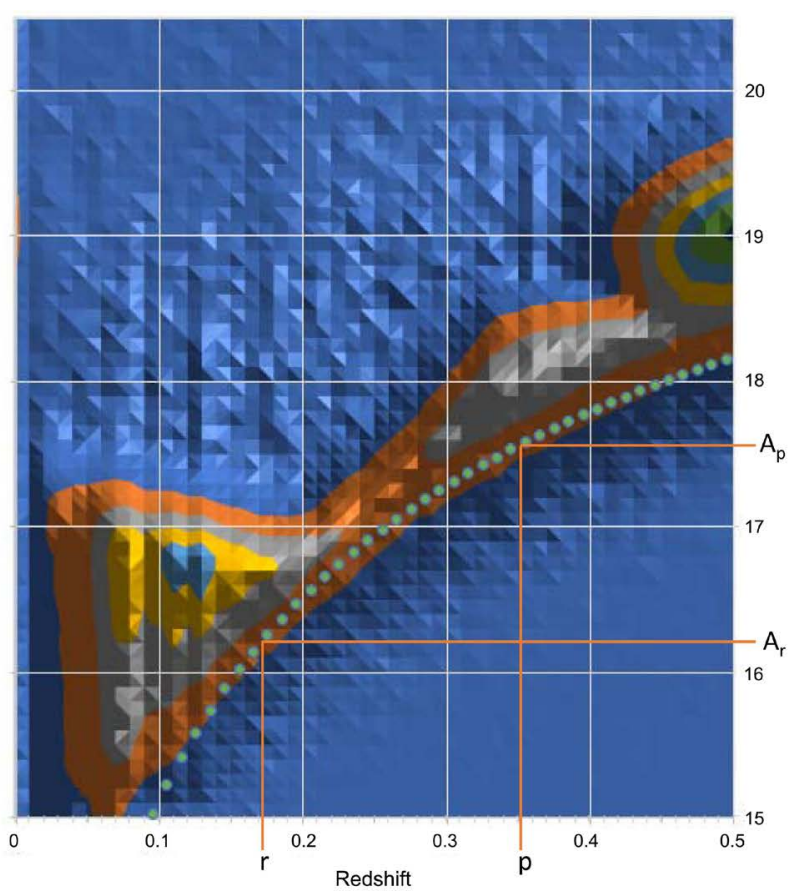

- $0-1000=1000-2000=2000-3000=3000-4000$

— $4000-5000=5000-6000 \simeq 6000-7000$

Figure 5. The distribution of measured luminosity as a function of the redshift. Magnitudes and redshifts were separated into bins of size $Z=0.1$ and $m=0.1$. 
- in the redshift range up to 0.5 , the vast majority of quasars have a lower luminosity than the brightest galaxies, contrary to the commonly held view that quasars are brighter than most galaxies.

Also,

- A chart (Figure 5, right hand chart) showing counts of galaxies against redshift-luminosity (apparent luminosity through the $\mathrm{z}$ filter, over 1.8 million galaxies included on this chart) shows that, at any redshift $r$ up to about 0.5 ,

- The galaxy counts are not significant at high luminosities, but become significant at a luminosity $A_{r}$ (e.g. in a redshift bin of 0.01 , and a luminosity bin of 0.1 , a count of 100 is significant when there are 1000 s of galaxies in the redshift bin) and

○ the galaxy counts increase with decreasing luminosity from $A_{r}$ until telescope detection limits reduce the counts (these limits are different at different redshifts as different telescopes and technologies have been used to find and measure the redshift and luminosities of galaxies) and

$\circ$ at a redshift $p$ that is greater than redshift $r$, the luminosity at the point $A_{p}$ (as defined for $A_{r}$ above) decreases with increasing redshift such that the relationship between $A_{r}$ and $A_{p}$ is as expected by the redshift-distance relationship proposed by Hubble (until telescope detection limits reduce the counts)

$\circ$ a dotted green line on the chart shows the expected redshift-distance relationship for a galaxy with absolute magnitude -22.9 .

- A similar chart (Figure 5, left hand chart, apparent luminosity measures using psfmag_z data, over 24 thousand quasars included on this chart) using data from quasars shows that, at any redshift $r$ from 0.03 up to about 0.2 ,

$\circ$ the quasar counts are not significant at higher luminosities, but become significant at a luminosity $B_{r}$ (e.g. in a redshift bin of 0.01 , and a luminosity bin of 0.1 , a count of 10 is significant when there are over 100 quasars in the redshift bin) and

○ the quasar counts increase with decreasing luminosity from $B_{r}$ and

$\circ$ quasar counts. in a redshift bin of 0.01 , and a luminosity bin of 0.1 , are not significant below a redshift of 0.04 ,

$\circ$ at a redshift $p$ that is greater than redshift $r$, the luminosity at the point $B_{p}$ decreases with increasing redshift, such that the relationship between $B_{r}$ and $B_{p}$ is not as expected by the redshift-distance relationship proposed by Hub$\underline{\text { ble }}$ and

$\circ$ in the redshift range 0.05 to 0.18 where telescope detection limits have minimal impact on the counts,

- after initially increasing from a luminosity $B_{p}$ to a luminosity $C_{p}$, the quasar counts then decrease to a luminosity $D_{p}$ where the counts become insignificant and

- the luminosity at $D_{p}$ (where redshift $p$ is greater than redshift $r$ ) decreases with increasing redshift such that the relationship between $B_{r}$ and $D_{p}$ is as expected by the redshift-distance relationship proposed by Hubble (until telescope detection limits at low luminosity reduce the counts). 
- The red dotted red line on the chart shows the expected Hubble redshift-distance relationship for an object with absolute magnitude of -19.2 , coincidentally (?) close to the absolute magnitude of type II supernovae, while the green dotted line shows the expected redshift-distance relationship for a galaxy with absolute magnitude -22.9 .

In summary, the key differences between the charts of galaxy and quasar counts are:

- the brightest significant galaxy counts at each redshift follow a line that reflects the Hubble redshift-distance relationship and other galaxies at that redshift are fainter,

- only the faintest significant quasar counts at any redshift in the range 0.02 to 0.06 follow a line that reflects the Hubble redshift-distance relationship.

This is a huge contradiction, yet it is clearly visible in the nearby universe where the redshift is less than 0.2 and quasar luminosity is greater than magnitude 18.5 where telescope detection limits do not have significant impact on the counts.

Looking at the quasar data in the redshift range $0<Z<0.2$ in more detail, a normalised data distribution curve of counts as a function of luminosity is found to be a close fit to the data in each redshift bin at all measured redshifts in the range $0.05<Z<0.20$. Hence, a normalised distribution curve was fitted to the data to determine the magnitude of the peak count for each redshift bin (Appendix $\mathrm{C}$ provides the charts of normalised data fitted to the quasar counts for psfmag_z measurements and the characteristics of the normalised data). The standard deviation around the peak magnitude is $0.4+/-0.05$ at a measured redshift of 0.05 , increasing to a standard deviation of $0.6+/-0.05$ at a measured redshift of 0.25 (above a redshift of 0.19 , assumptions were made about the reduction in counts occurring from about psfmag $z=18.5$ due to telescope detection limits). Most quasars ( $>80 \%$ ) fall within the normalised distribution in each of these redshift bins.

As counts of quasars in the redshift range $0<Z<0.2$ and luminosity range 15 $<$ psfmag_z $<18.5$ have minimal impact from telescope detection limits, the normal distribution of luminosity for quasars appears to be a significant finding.

Might many quasars not follow the expected redshift-distance relationship? Might the luminosity of quasars follow a normalised distribution? In the following sections, these anomalies in the quasar counts are investigated further and two proposals are presented that might explain why the SDSS data for quasar counts is different from the chart showing galaxy counts. Astronomers and cosmologists are invited to investigate these proposals and others until a clear explanation of the quasars counts at redshifts less than 1.0 is found.

The following sections cover:

1) an interpretation of the quasar counts that assumes all quasars are at the distance indicated by Hubble's law;

2) a proposal that could explain the quasar counts if quasars have an inherent blueshift in addition to a redshift that is associated with the distance to the qua- 
sar. This proposal also requires quasars to have a narrow luminosity range, with a normal distribution around a peak luminosity.

3) a proposal that could explain the quasar counts if quasars have an inherent redshift in addition to a redshift that is associated with the distance to the quasar. This proposal also requires quasars to have a narrow luminosity range and the quasars with the lowest luminosity at a redshift would be the most luminous at that distance, with other quasars at that distance having higher redshifts!!

Each of these interpretations of the quasar counts has anomalies in them. They are presented here to stimulate discussions as to the true explanation of quasar counts and provoke further research into quasar counts at relatively low $(Z<0.5)$ redshift.

\section{Are Quasars Truly at the Distance Indicated by Hubble's Law?}

The quasar counts start becoming significant at about a redshift of 0.03 and a measured luminosity (psfmag_z) of about 16.0. This equates to an absolute magnitude of about -19.2. However, as redshift increases, the quasar counts start becoming significant at lower luminosity levels (i.e. quasars are brighter). For example, at a redshift of 0.2 , quasar counts start becoming significant at a measured luminosity (psfmag_z) of about 17.3, which equates to an absolute magnitude of about -22.2 . Note that quasar counts increase with higher redshifts, so the count at which quasar counts are considered to be significant needs to be increased. At a redshift of 0.2 , the correction leads to quasar counts start becoming significant at a measured luminosity (psfmag_z) of about 17.6, which equates to an absolute magnitude of about -21.9. Hence, this equates to an increase in luminosity of about 0.7 magnitude. Do quasars really increase in brightness the further back we look in the universe? The lookback time to a redshift of 0.03 where this starts happening is only about 500 million years, yet it appears quasars have been evolving up to that point. Is that believable?

Quasar counts in the redshift range $0.05<Z<0.25$ exhibit a peak count that appears natural i.e. it has not been created by telescope detection limits. However, the peak counts do not follow Hubble's redshift-distance relationship. Why not?

\section{Do Quasars Have an Inherent Blueshift?}

The data from the charts in Appendix $\mathrm{C}$ and similar charts was used to generate the charts in Figure 6. The charts in Figure 6 show the predicted peak psfmag_z magnitude at various redshift, based on the normalised luminosity distribution curve that was fitted to the SDSS data. A line of best fit to the data was generated and is also shown on the charts. This line represents a Hubble redshift-distance relationship, with $H=70 \mathrm{~km} / \mathrm{sec} /$ Megaparsec. In order to obtain the best fit to the data points, a blueshift has to be added to all points. The characteristics of the best fit lines show that, for quasars: 

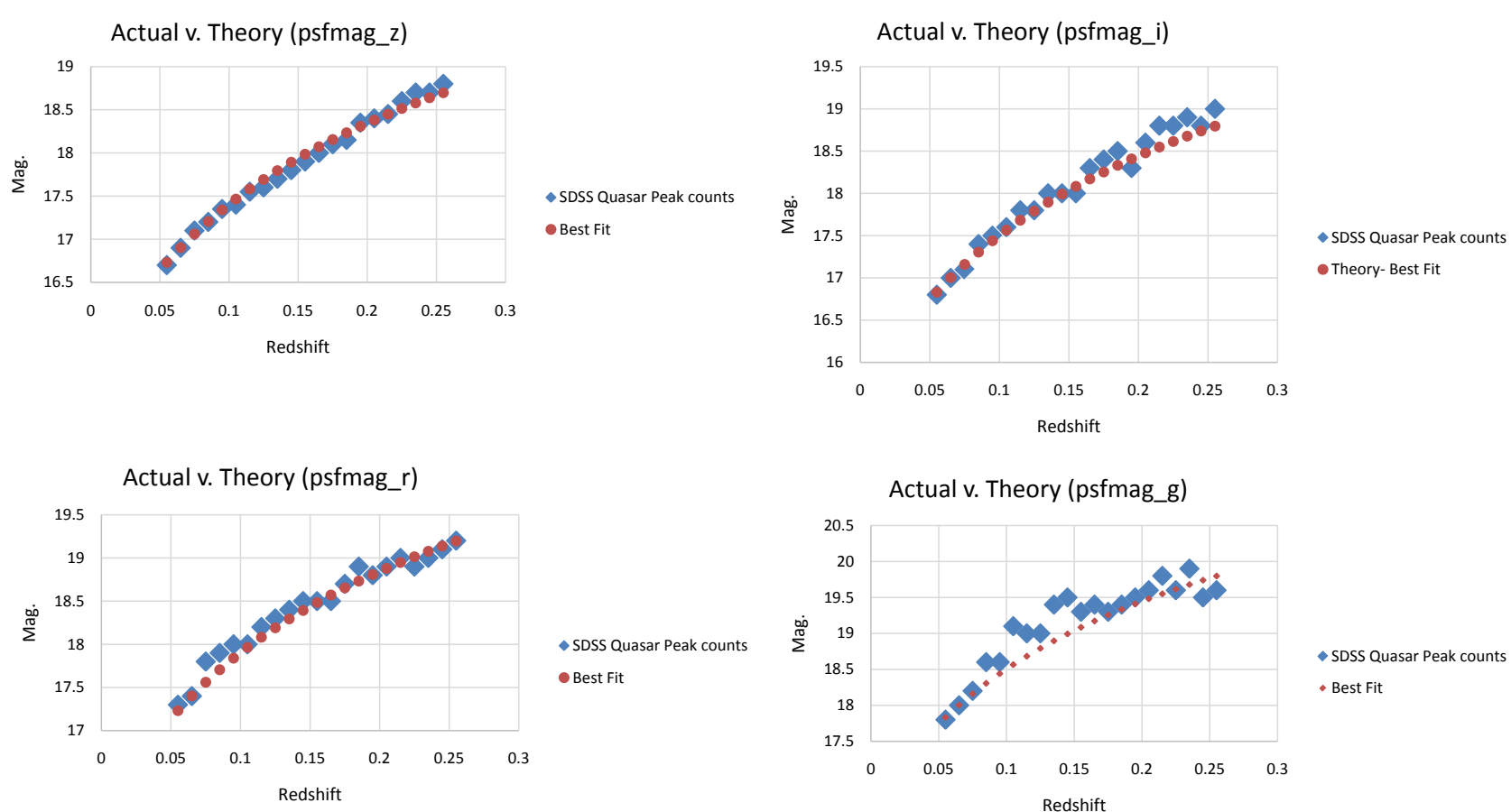

Figure 6. Count of quasars: measured redshift v. luminosity.

- the luminosity is related to measured redshift plus a blueshift of $18,000+/-$ $5000 \mathrm{~km} / \mathrm{sec}$.

- quasars at the peak luminosity have an absolute magnitude of $-21.6+/-0.2$.

The accuracy of the luminosity measurement of peak count at any redshift in this range is $+/-0.1$. There were no significant counts found through the $\mathrm{u}$-filter, where luminosity is lowest. A peak count in the $\mathrm{z}$ filter at, say, redshift 0.05 requires a quasar in that count to be at a distance equivalent to a redshift of 0.12 for it to have a luminosity that fits the proposed blueshift correction.

To create the line of best fit, a redshift-distance relationship was calculated using the formula ([9]) i.e. redshift is assumed to originate from a velocity of recession):

$$
\text { Distance }=c *\left(\left((Z+1)^{2}-1\right) /\left((Z+1)^{2}+1\right)\right) / H
$$

where $c$ is the velocity of light, $Z$ is the true redshift of the quasar, corrected from the measured redshift with the blueshift mentioned above, and $H$ is Hubble's constant $(70 \mathrm{~km} / \mathrm{sec} /$ Megaparsec).

The absolute magnitude of quasars at the peak counts is:

- -21.6 through the $\mathrm{z}$ filter,

- -21.5 through the i filter,

- -21.1 through the $r$ filter,

- -20.5 through the g filter.

A best fit normalised distribution was found for each redshift bin (see Appendix C) and cumulative best fit counts within a redshift bin were compared against the actual count. In addition, the counts were compared against the vo- 
lume of space in which the counts are being made (see Figure 7 for details). The resulting chart shows that actual counts were found to increase linearly with redshift up to a measured redshift of 0.2 , after which there is a reduction in actual counts at luminosity of about psfmag_z $z=19.0$, presumably due to the SDSS technology not operating efficiently at this level.

While the proposed blueshift and the normalised luminosity distribution appear to justify the quasar counts at low redshift, the redshift range over which these results are found is relatively small. Do quasars at higher redshift follow normalised curves and have blueshifts too? Are all quasars further away than we have believed based on years of research? Why would all quasars have a specific blueshift?

\section{Do Quasars Have an Inherent Redshift?}

A key finding of the charts showing the distribution of quasar counts is that in the redshift in the range 0.03 to 0.08 :

- after initially increasing to a peak count as luminosity decreases, the quasar counts then decrease to a luminosity (see the point $D_{p}$ on Figure 5) where the counts become insignificant, and

- the luminosity of the quasars in these specific counts decreases with increasing redshift according to the redshift-distance relationship proposed by Hubble (until telescope detection limits at low luminosity reduce the counts).

While this is a small redshift range, telescope detection limits at low luminosity reduce the counts at higher redshift, so it is currently not possible to prove if this relationship continues at higher redshifts.

To explain this aspect of the counts, it is proposed that, while every quasar has a redshift that originates from the redshift due to its distance from Earth, many quasars have an additional, inherent redshift. The additional redshift could originate from quasars having either, or both:

1) a gravitational redshift, bearing in mind the light from a quasar comes from a very small source with the properties of a huge black hole or,

\section{Counts of quasars by redshift}

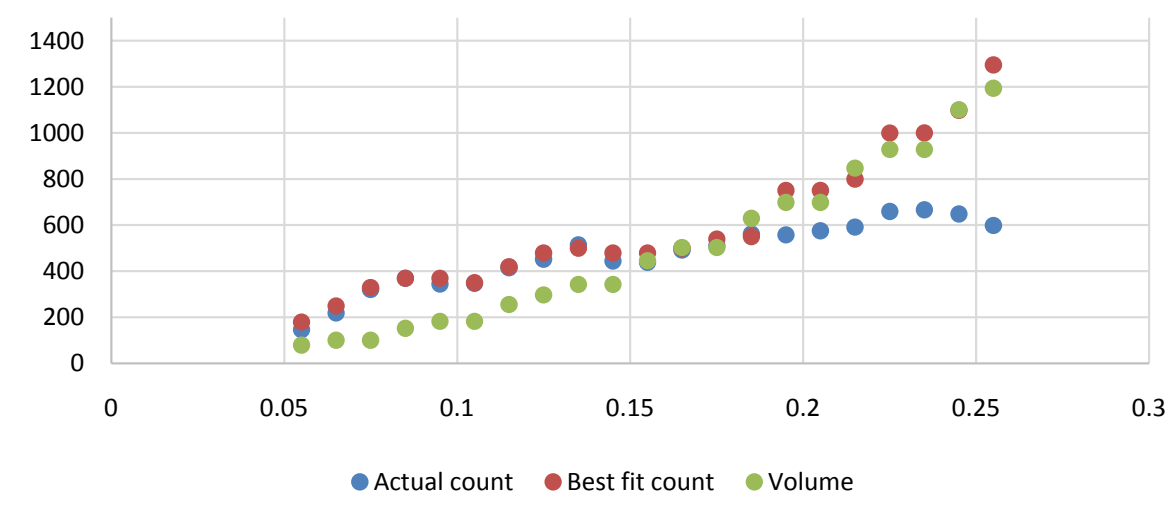

Figure 7. Count of quasars by measured redshift. 
2) a surrounding cloud of electrons, protons, atoms etc. that

a) reduce the luminosity of quasars and

b) increase the redshift that we measure for the quasar.

Considering the quasar cloud a little further: photons passing through such a cloud would interact with electrons frequently and they would lose energy in small increments, probably through the Compton effect (delta $\lambda=\left(h / m_{e} \mathcal{c}\right)(1-$ $\cos \theta)$ ) and/or possibly also through "New Tired Light" processes ([10] [11] [12] and [13]). The wider the cloud and/or the denser it is, the greater redshift would be seen. Note that the density of the cloud must be low enough such that the photons interacting with electrons do not result in the emerging photons appearing as photons from a black body.

Such clouds would probably evolve slowly with time as protons and electrons combined into hydrogen, etc. Note that protons could also be in such a cloud in equal numbers to the electrons, but their mass is so much larger that their impact on redshift (e.g. due to the Compton effect) would be negligible.

The impact of this proposal on the quasar counts that we measure is probably best explained with reference to a chart (see Figure 8). Consider initially only a set of quasars that are all at a distance $D_{1}$ such that each quasar has a redshift $R_{D 1}$ that is related to the distance $D_{1}$ by Hubble's law. The proposal is that:

- each quasar is surrounded by a shell of electrons, protons, atoms etc. and each shell will have a different thickness and density.

- the brightest quasar at a distance will have the thinnest shell around it and a luminosity $L_{D 1}$; all other quasars at distance $D_{1}$ will be dimmer due to absorption/dissipation in the shell.

- all quasars are inherently the same with the same source luminosity; the only difference between them is the size/density of the shell of particles around them.

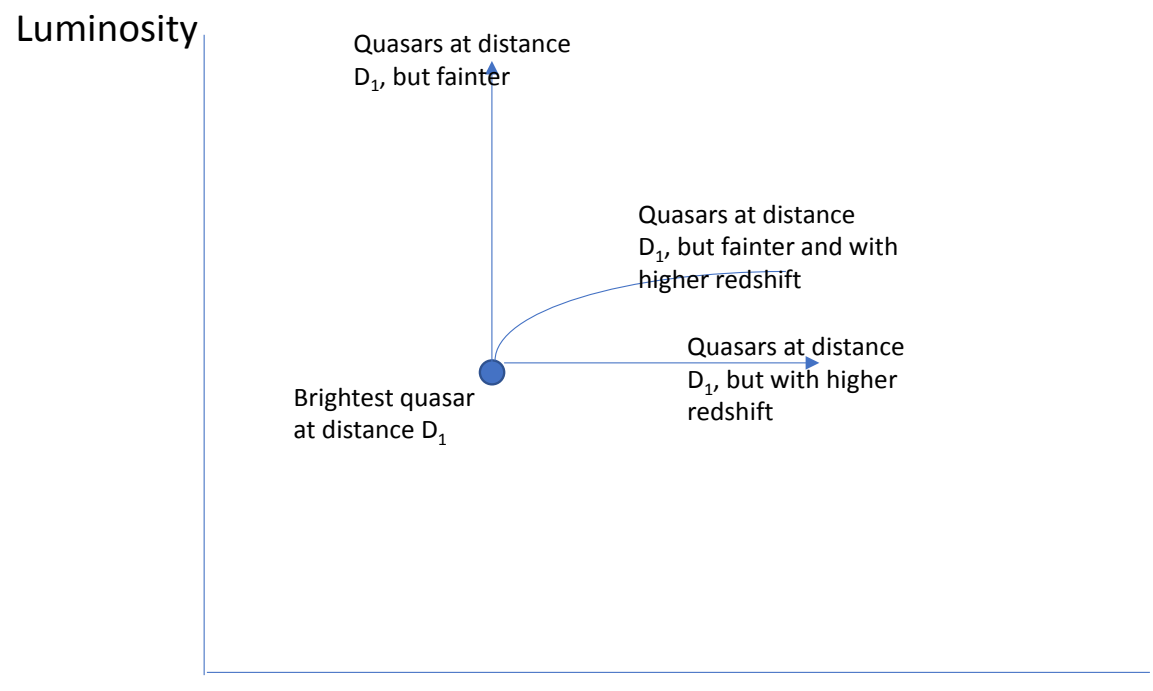

Redshift

Figure 8. Proposed redshift-luminosity relationship for quasars. 
- most quasars will be observed to have an additional redshift related to the thickness/density of the shell around it, so their measured redshift will be greater than $R_{D 1}$.

Note that a quasar's shell might be in the form of a torus, so our line of sight to the quasar would affect the quantity of the shell between the quasar and us, and therefore the brightness and redshift as described here.

Repeating this description for many distances $D_{n}$ leads to the following chart (Figure 9). Converting this chart to a quasar count against redshift and luminosity chart could result in a chart that is close to what we see with the SDSS data. Note that the leading edge of the chart (highlighted in red) would follow the Hubble redshift-distance law, which is what we see in the SDSS data.

Looking at the SDSS data for the brightest quasars where the redshift is about 0.03 (luminosity $L_{0.03}$ is about mag 16 ), if the above proposal applies, then the SDSS data indicates that, at a distance where the base redshift is 0.03 , most of the quasars have higher redshifts and are about 1.6 magnitude fainter. The few quasars that are close to having luminosity $L_{0.03}$ have low redshift increments over $R_{D 1}$ and low luminosity reduction

If this proposal is correct, then almost all the quasars that we can see (several hundred thousand) have a base redshift of less than 0.5 , so they are more frequently found in the relatively nearby universe than currently thought. It also means that most of the quasars we see with magnitude 18.0 (i.e. the brightest quasars at most redshifts) originate from quasars with true/basic redshift of 0.03 . That is a lot of quasars (about 3500 at mag. 18.0), with similar luminosity, yet very different redshifts (note that there are tens of thousands of galaxies at this luminosity, so 3500 is not such a surprising number).

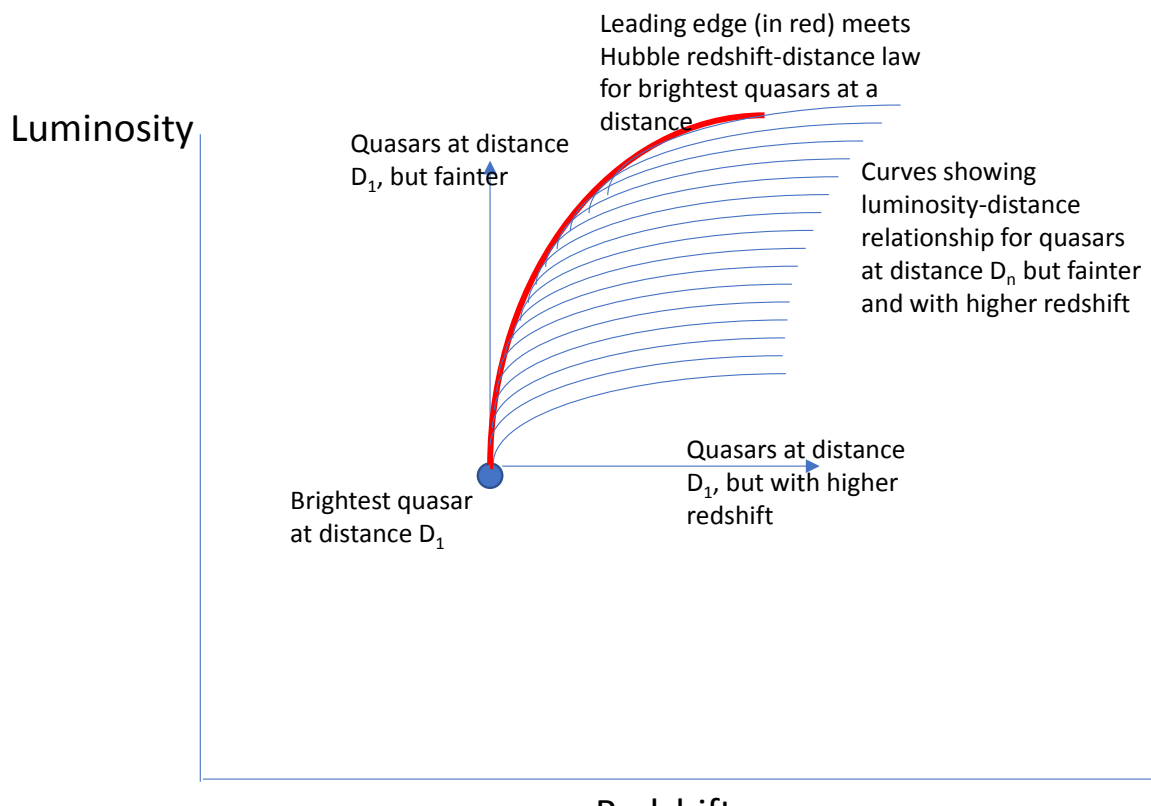

\section{Redshift}

Figure 9. Redshift-luminosity relationship for quasars at many distances greater than $D_{1}$. 
The peak count of quasars is at mag. 19.6 where the SDSS survey has found about 15,600 quasars. Those quasars (according to my proposal) would largely originate from quasars at a distance with a base redshift of 0.07 . Put another way, about $2 / 3$ of the quasars from the 240,000 quasars used to form the chart fall in the range 0.03 to 0.07 . In the range 0.03 to 0.07 there are about 245,000 galaxies in the SDSS survey galaxy counts. Is it a coincidence that there are a similar number of galaxies to the quasars counts in the same redshift range?

If there is a cloud of electrons around a quasar, what might the mass of this cloud be? The following is not a proof, but is given as an indication of what the mass of the cloud might be.

Quasars' sizes have been measured as being approximately 1 to 2 light year across. Consider a photon passing through such a cloud of electrons and protons:

1) A photon has a collision cross-section with an electron defined by $\sigma=2 r \lambda$, where $r$ is the classical electron radius $2.82 \times 10^{-15} \mathrm{~m}$.

2) The mean free path of a photon through a quasar's shell that is filled only with electrons is $I=1 / n \sigma$, where $n$ is the density of electrons in the shell.

3) During its travel through a shell of radius $D$, photons will have $D / I=D n \sigma$ interactions with electrons.

4) On each photon-electron interaction,

o the wavelength of the transmitted photon is increased by an amount $h / m c=$ $2.43 \times 10^{-12} \mathrm{~m}$ (the Compton constant) from the incoming photon regardless of the wavelength of the incoming photon.

- The above applies to low energy photons (e.g. visible spectrum at wavelength $5 \times 10^{-7} \mathrm{~m}$ ) and not to high energy (e.g. X-rays at wavelength $10^{-10} \mathrm{~m}$ ) photons where relativistic effects occur.

- A photon -proton interaction will have negligible impact on the wavelength of the transmitted photon as the mass of the proton is significantly higher than that of an electron.

5) The total increase in wavelength over $D$ will be $D n \sigma^{\star} h / m c=D n 2 r \lambda h / m c$.

6) Redshift $z=\delta \lambda / \lambda$. For small $z, z=D n 2 r \lambda h / m c \lambda=2 n r h D / m c$. For large $z, z$ $=\exp (2 n r h D / m c)-1$. Hence, $\ln (z+1)=2 n r h D / m c, n=m \ln (z+1) / 2 r h D$.

7) So, to have an additional redshift of 1 , the density of the electron cloud would be $n=\ln (1+1) /\left(2 \times 2.82 \times 10^{-15} \times 2.43 \times 10^{-12} \times 3 \times 10^{8} \times 3 \times 10^{7}\right)=5.6$ $\times 10^{9}$ electrons $/ \mathrm{m}^{3}$, or about 5 electrons per cubic $\mathrm{mm}$.

In summary, an electron cloud that extends one light year from a quasar would need to have a density of 5 electrons per cubic $\mathrm{mm}$ in order to create a redshift of 1 in the light leaving the quasar.

Such an electron cloud would have a volume of $V=4 \pi r^{3} / 3=4 \pi\left(3 \times 10^{8} \times 3 \times\right.$ $\left.10^{7}\right)^{3} / 3=9.2 \times 10^{48} \mathrm{~m}^{3}$

If the cloud is a mixture of protons and electrons, its mass would be

$=$ density ${ }^{*}$ volume ${ }^{*}$ mass-of-proton

$=4.2 \times 10^{9} \times 9.2 \times 10^{48} \times 1.7 \times 10^{-27}=6.6 \times 10^{31} \mathrm{~kg}$ 
Our sun's mass is $2 \times 10^{30} \mathrm{~kg}$, so the cloud's mass is equivalent to 30 suns. While that may seem a lot, a quasar's luminosity is thought to be equivalent to galaxies with billions of suns, so a cloud of this mass is not unrealistic in relation to the quasar.

While this proposal appears to justify the quasar counts, there is strong evidence from many sources that there are many quasars that are highly luminous and at large distances indicated by Hubble's law for their high redshift. How do they relate to this proposal? Also, this proposal does not explain why we see peaks in quasar counts in the redshift range 0.05 to 0.25 without adding some complexity to the proposal.

\section{Conclusions}

While there is insufficient evidence to determine why the counts of quasars result in the charts presented here, there is clear evidence that, at least for thousands of quasars at low redshift where we should be able to determine their characteristics most accurately, there are unexpected anomalies in the distribution of quasar counts. Specifically,

1) Quasar counts do not follow the expected redshift-distance relationship.

2) The majority of quasars at low redshift have a luminosity that is less than the brightest galaxies, whereas at high redshift, the majority of quasars we see have a higher luminosity than galaxies.

3) The luminosity distribution of quasars follows a normal distribution.

The evidence from studies of galaxies and supernovae indicates that quasars should have an element of their redshift that follows Hubble's law. However, there are clearly additional factors affecting the redshift of quasars. This paper presents a couple of proposals that could explain the distribution of quasar counts i.e.

1) Quasars have an inherent blueshift of $18,000+/-5000 \mathrm{~km} / \mathrm{sec}$ in their redshift. Might this originate from a symmetrical outflow of gas from the quasars? Is the true distance to quasars further than the measured redshift indicated?

2) Most quasars have an inherent redshift and are relatively close to Earth, with redshifts less than 1 . Are they surrounded by a cloud that introduces an additional redshift to their cosmological redshift?

Astronomers are urged to find more quasars in the redshift range up to a redshift of 0.5 in order to clarify why quasars do not follow the same redshift-distance relationship as measured for galaxies.

\section{Acknowledgements}

Funding for the Sloan Digital Sky Survey IV has been provided by the Alfred P. Sloan Foundation, the U.S. Department of Energy Office of Science, and the Participating Institutions. SDSS acknowledges support and resources from the Center for High-Performance Computing at the University of Utah. The SDSS web site is http://www.sdss.org/. 
SDSS is managed by the Astrophysical Research Consortium for the Participating Institutions of the SDSS Collaboration including the Brazilian Participation Group, the Carnegie Institution for Science, Carnegie Mellon University, the Chilean Participation Group, the French Participation Group, Harvard-Smithsonian Center for Astrophysics, Instituto de Astrofísica de Canarias, The Johns Hopkins University, Kavli Institute for the Physics and Mathematics of the Universe (IPMU)/University of Tokyo, the Korean Participation Group, Lawrence Berkeley National Laboratory, Leibniz Institut für Astrophysik Potsdam (AIP), Max-Planck-Institut für Astronomie (MPIA Heidelberg), Max-Planck-Institut für Astrophysik (MPA Garching), Max-Planck-Institut für Extraterrestrische Physik (MPE), National Astronomical Observatories of China, New Mexico State University, New York University, University of Notre Dame, Observatório Nacional/MCTI, The Ohio State University, Pennsylvania State University, Shanghai Astronomical Observatory, United Kingdom Participation Group, Universidad Nacional Autónoma de México, University of Arizona, University of Colorado Boulder, University of Oxford, University of Portsmouth, University of Utah, University of Virginia, University of Washington, University of Wisconsin, Vanderbilt University, and Yale University.

\section{Conflicts of Interest}

The author declares no conflicts of interest regarding the publication of this paper.

\section{References}

[1] Lyke, B.W., Higley, A.N., McLane, J.N., Schurhammer, D.P., Myers, A.D., Ross, A.J., et al. (2020) The Sloan Digital Sky Survey Quasar Catalog: Sixteenth Data Release. https://arxiv.org/pdf/2007.09001.pdf

[2] Stoughton, C., Lupton, R.H., Bernardi, M., Blanton, M.R., Burles, S., Castander, F.J., et al. (2002) Sloan Digital Sky Survey: Early Data Release. The Astronomical Journal, 123, 485-548. https://doi.org/10.1086/324741

[3] Humason, M.L., Mayall, N.U. and Sandage, A.R. (1956) Redshifts and Magnitudes of Extragalactic Nebulae. Astronomical Journal, 61, 97-162. https://doi.org/10.1086/107297

[4] Oke, J.B. and Sandage, A. (1968) Energy Distributions, K Corrections, and the Stebbins-Whitford Effect for Giant Elliptical Galaxies. Astrophysical Journal, 154, 21. https://doi.org/10.1086/149737

[5] Peterson, B.M. (1997) An Introduction to Active Galactic Nuclei. Cambridge University Press, Cambridge. https://doi.org/10.1017/CBO9781139170901

[6] Hogg, D.W., Baldry, I.K., Blanton, M.R. and Eisenstein, D.J. (2002) The K Correction. arXiv: astro-ph/0210394

[7] Youll, D. (2019) Anomalies in the Redshifts of Quasars Using SDSS DR14 Data. https://www.researchgate.net/publication/335883111 Anomalies in the redshifts of Quasars using SDSS DR14 data

[8] Youll, D. (2019) Anomalies in the Redshifts of SDSS DR14 Galaxy Data. https://www.researchgate.net/publication/333532525 Anomalies in the redshifts 
of SDSS DR14 Galaxy data

[9] Hogg, D.W. (2000) Distance Measures in Cosmology. arXiv: astro-ph/9905116 https://arxiv.org/abs/astro-ph/9905116v4

[10] Ashmore, L.E. (2006) Recoil between Photons and Electrons Leading to the Hubble Constant and CMB. Galilean Electrodynamics, 17, 53-60.

[11] Ashmore, L.E. (2016) An Explanation of Redshifts in a Static Universe. In: Amoroso, R.L., Kauffman, L.H. and Rowlands, P., Eds., Unified Field Mechanics, World Scientific Publishers, Singapore, 456-462. https://doi.org/10.1142/9789814719063 0046

[12] Ashmore, L.E. (2016) A Relationship between Dispersion Measure and Redshift Derived in Terms of New Tired Light. Journal of High Energy Physics, Gravitation and Cosmology, 2, 512-530. https://doi.org/10.4236/jhepgc.2016.24045

[13] Trinchera, A. (2021) Redshift Anomaly on the Solar Disk as Multiple Interactions between Photons and Electrons. Journal of High Energy Physics, Gravitation and Cosmology, 7, 1-51. https://doi.org/10.4236/jhepgc.2021.71001

[14] York, D.G., Adelman, J., Anderson Jr., J.E., Anderson, S.F., Annis, J., Bahcall, N.A., et al. (2000) The Sloan Digital Sky Survey: Technical Summary. The Astronomical Journal, 120, 1579-1587. https://doi.org/10.1086/301513

[15] Abazajian, K.N., Adelman-McCarthy, J.K., Agüeros, M.A., Allam, S.S., Allende Prieto, C., An, D., et al. (2009) The Seventh Data Release of the Sloan Digital Sky Survey. The Astrophysical Journal Supplement Series, 182, 543-558. https://doi.org/10.1088/0067-0049/182/2/543

[16] Eisenstein, et al. (2011) The SDSS-III Baryon Oscillation Spectroscopic Survey: Quasar Target Selection for Data Release Nine. Astrophysical Journal Supplement Series, $199,3$.

[17] Ross, et al. (2012) The Composite Spectrum of BOSS Quasars Selected for Studies of the Lyman-a Forest. The Astrophysical Journal, 151, 155. 


\section{Appendix A: Distribution of Quasar Luminosities}

The counts at redshifts from 0.05 to 0.25 (see Appendix C for details) indicate that the profile of the counts of quasars follows a normal (Gaussian) distribution, with minimal impact on counts due to flux limits of the detectors. Hence, a normal distribution curve was fitted to the counts of quasars to determine the characteristics of the normal distribution.

If the distance to a quasar is truly related to its redshift according to Hubble's law, then the distance to the quasar can be calculated using the formula:

$$
\text { Distance }=c *\left(\left((Z+1)^{2}-1\right) /\left((Z+1)^{2}+1\right)\right) / H
$$

Figure A1 shows the expected profile of the count of quasars at two redshifts, with distance calculated using the above equation and counts reflecting the volume of space in which the counts are made and no correction made for counts at low luminosity being reduced due to the difficulty in detecting quasars at low luminosity. Note that quasar counts in the SDSS DR16 data start dropping from their predicted values at a luminosity of about 19.0. Hence, fitting normalised distribution profiles to quasar counts becomes inaccurate above a measured redshift of about 0.25 .

\section{Expected Count v. Luminosity at two redshifts}

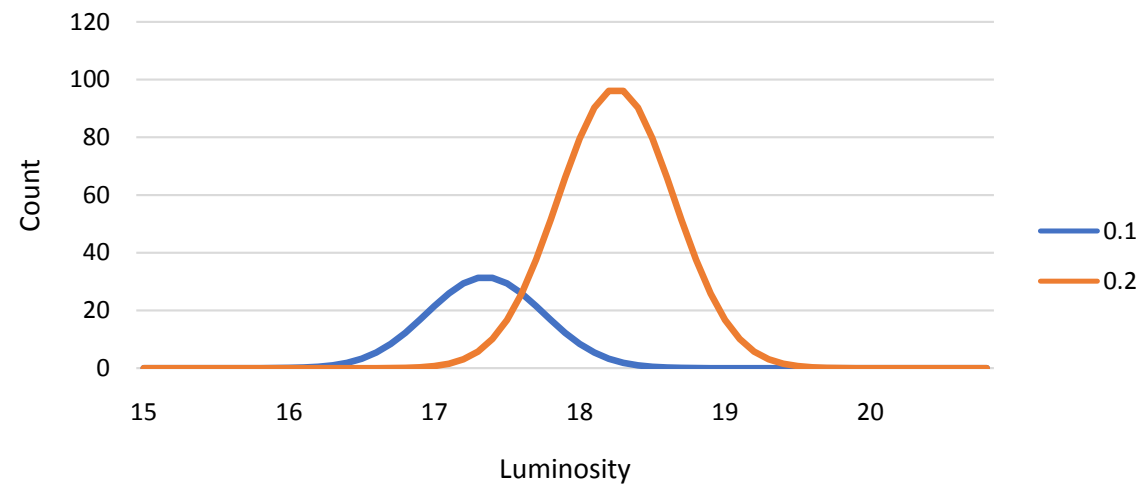

Figure A1. Possible profile of quasar counts at different distances.

\section{Appendix B: The SDSS Survey and Quasar Selection}

Large redshift surveys were initiated by the Sloan Digital Sky Survey (SDSS) ([14]) initially to measure redshifts of nearly one million galaxies in spectroscopic observations between 2000 and 2008 (the phases known as SDSS-I and -II, described in the seventh data release, DR7, by [15]). The SDSS-I and -II imaging programs provide two large, contiguous regions of sky that lie away from the Galactic plane.

The Baryon Oscillation Spectroscopic Survey (BOSS) is the largest of the four surveys that comprise SDSS-III ([16]). BOSS was designed to measure the scale of baryon acoustic oscillations (BAO) in the clustering of matter over a larger volume than the combined efforts of all previous spectroscopic surveys of 
large-scale structure.

The BOSS quasar survey pioneered a novel method of measuring $\mathrm{BAO}$ at high redshift $(2.15<z<3.5)$ using Lyaforest absorption towards a dense grid of background quasars. The redshifted Ly $\alpha$ line becomes detectable in the BOSS spectral range just beyond $z=2$ and becomes highly opaque at $z>4$, motivating the targeted redshift range.

An average quasar spectrum and how a quasars colours evolve with redshift is described by [17].

\section{Appendix C: Fits of Normalised Curves to the Actual Counts against Redshift}

Table $\mathrm{C} 1$ gives the characteristics of the best fit normal distributions to the counts of quasars against psfmag_z luminosity for each redshift bin. The best fit normal distributions v. actual counts are shown in Figure C1.

Table C1. Normal distribution parameters to fit SDSS DR16 quasar data.

\begin{tabular}{|c|c|c|c|c|}
\hline Measured & Actual count & Best fit count & SD & Peak mag \\
\hline 0.05 & 147 & 180 & 0.43 & 16.7 \\
\hline 0.06 & 219 & 250 & 0.4 & 16.9 \\
\hline 0.07 & 321 & 330 & 0.4 & 17.1 \\
\hline 0.08 & 370 & 370 & 0.4 & 17.2 \\
\hline 0.09 & 345 & 370 & 0.4 & 17.35 \\
\hline 0.1 & 348 & 350 & 0.4 & 17.35 \\
\hline 0.11 & 415 & 420 & 0.43 & 17.55 \\
\hline 012 & 452 & 800 & 0.45 & 17.6 \\
\hline 0.13 & 515 & 500 & 0.45 & 17.7 \\
\hline 0.14 & 444 & 480 & 0.45 & 17.8 \\
\hline 0.15 & 439 & 480 & 0.5 & 17.9 \\
\hline 0.16 & 492 & 500 & 0.47 & 18 \\
\hline 0.17 & 511 & 540 & 0.5 & 18.1 \\
\hline 0.18 & 562 & 550 & 0.5 & 18.15 \\
\hline 0.19 & 558 & 750 & 0.55 & 18.35 \\
\hline 0.2 & 575 & 750 & 0.6 & 18.4 \\
\hline 0.21 & 592 & 800 & 0.6 & 18.55 \\
\hline 0.22 & 659 & 1000 & 0.6 & 18.6 \\
\hline 0.23 & 666 & 1000 & 0.65 & 18.7 \\
\hline 0.24 & 648 & 1098 & 0.7 & 18.8 \\
\hline 0.25 & 599 & 1295 & 0.7 & 19 \\
\hline
\end{tabular}




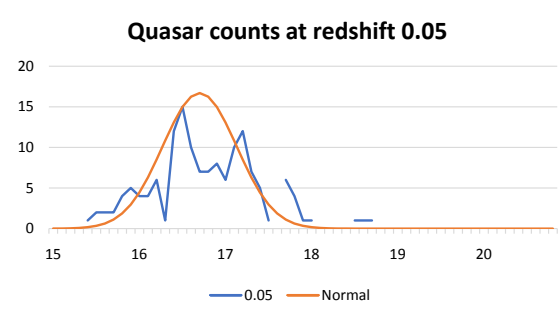

Quasar counts at redshift 0.08

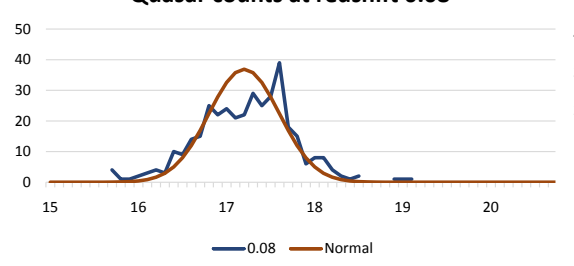

Quasar counts at redshift 0.11

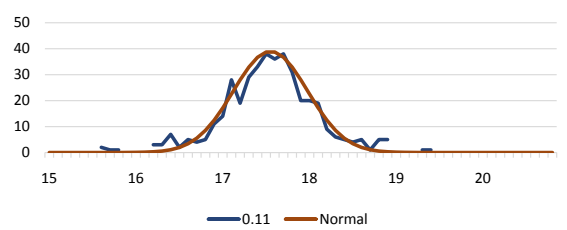

Quasar counts at redshift 0.14

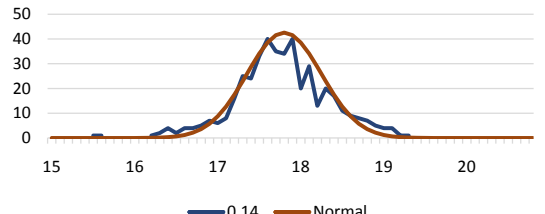

Quasar counts at redshift 0.17

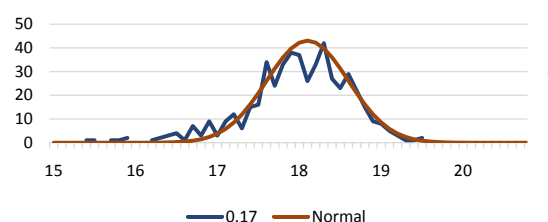

Quasar counts at redshift $\mathbf{0 . 2 0}$

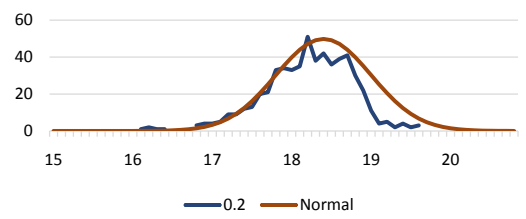

Quasar counts at redshift $\mathbf{0 . 2 3}$

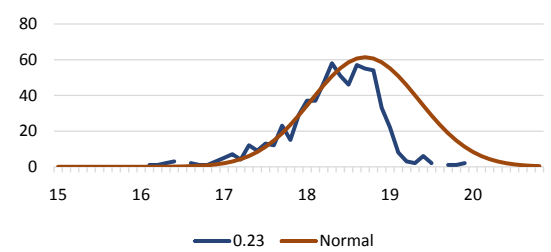

Quasar counts at redshift 0.06

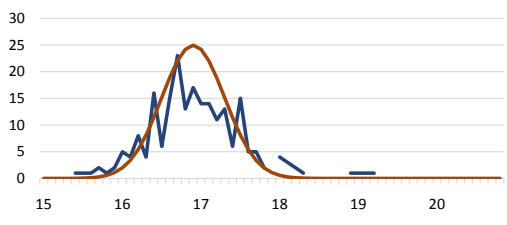

$-0.06-$ Normal

Quasar counts at redshift 0.09

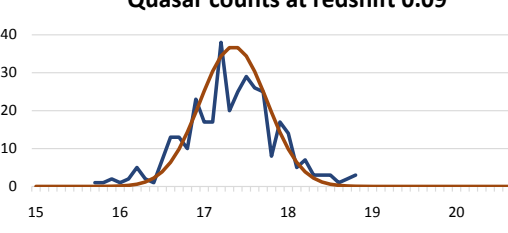

- $0.09-$ Normal

Quasar counts at redshift $\mathbf{0 . 1 2}$

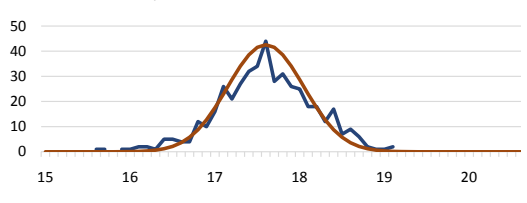

- 0.12 - Normal

Quasar counts at redshift $\mathbf{0 . 1 5}$

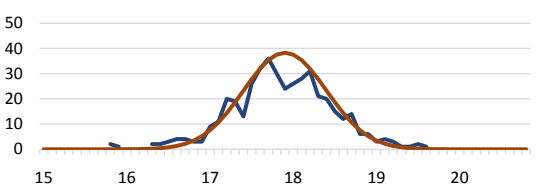

-0.15-Normal

Quasar counts at redshift 0.18

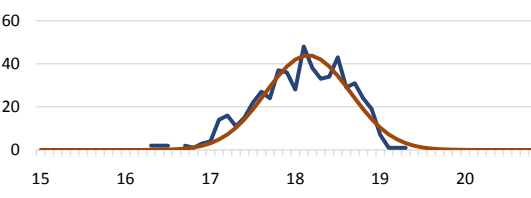

$$
\text { -0.18-Normal }
$$

Quasar counts at redshift $\mathbf{0 . 2 1}$

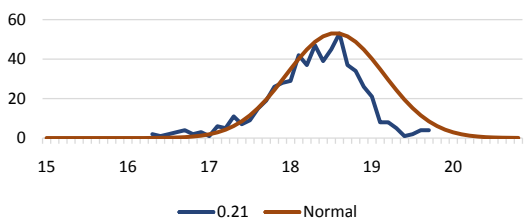

Quasar counts at redshift $\mathbf{0 . 2 4}$

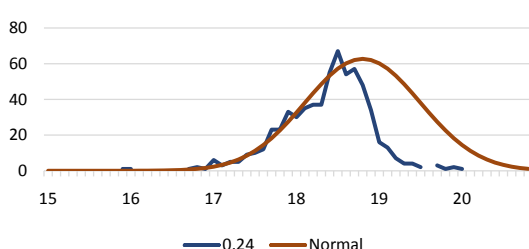

Quasar counts at redshift 0.07

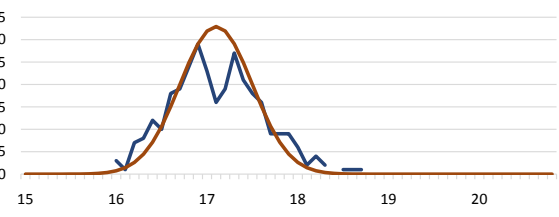

- $0.07-$ Normal

Quasar counts at redshift $\mathbf{0 . 1 0}$

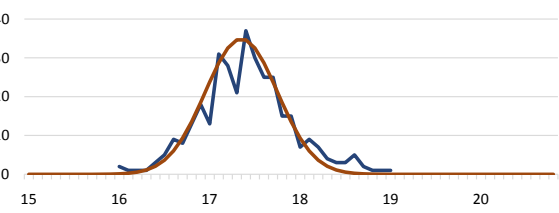

- 0.1 - Normal

Quasar counts at redshift $\mathbf{0 . 1 3}$

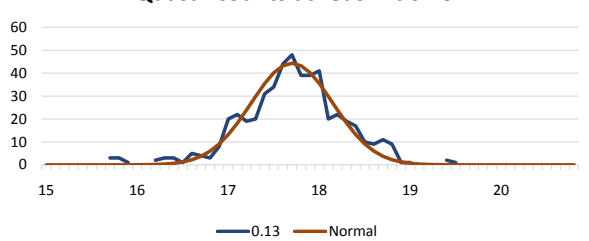

Quasar counts at redshift $\mathbf{0 . 1 6}$

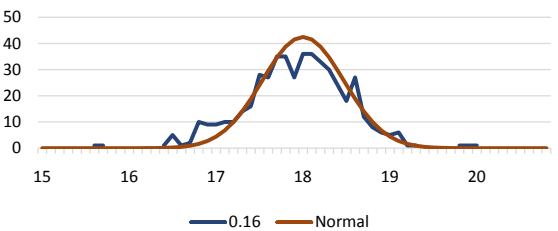

Quasar counts at redshift 0.19

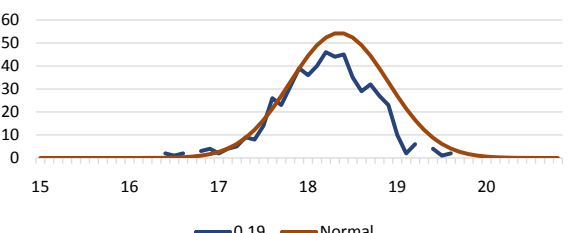

Quasar counts at redshift $\mathbf{0 . 2 2}$

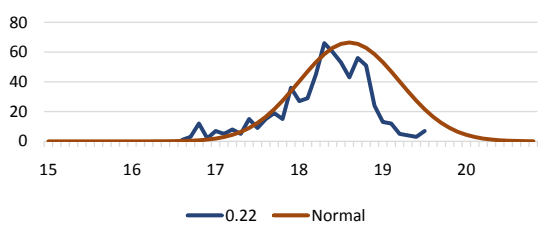

Quasar counts at redshift $\mathbf{0 . 2 5}$

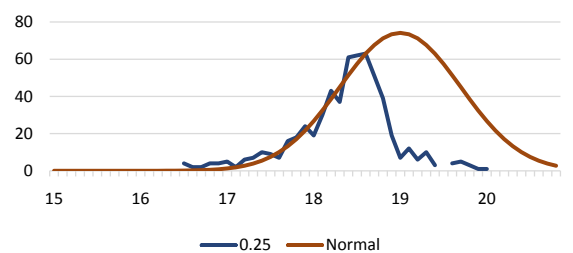

Figure C1. Best fit normal distributions v. actual counts of quasars. 